\title{
The Effect of Ginkgo biloba Extracts on Candida albicans Isolated from Healthy Persons Zahraa S. Qasim*,1
}

\author{
*Department of Clinical Laboratory Science, College of Pharmacy, University of Mosul, Mosul, Iraq.
}

\begin{abstract}
The objective was to study the effect of prepared Ginkgo biloba (as aqueous and ethanolic) extracts against Candida albicans isolated from healthy persons. After isolation and identification of C.albicans, conduct 4 tests; susceptibility test, biofilm formation test, phytochemical screening test, and antioxidant activity test. One hundred oral swabs sample were obtained from healthy persons with oral lesion attending dentistry teaching hospital in Dentistry College, their age ranged from 1-30 years of both sexes. The studied samples collected through 8 months (April - December / 2018). From 100 healthy person involved in this study, there were 21(21\%) C. albicans isolates revealed from clinical specimens. Zone of inhibition for C. albicans was higher in ethanol than aqueous extracts. Three (14.2\%) isolates showed positive biofilm formation in tube method, phytochemical reaction in ethanol extract showed 5 phytochemical compounds, while aqueous extract showed 4 phytochemical compounds, in addition to antioxidant activity in ethanol extract was higher than aqueous. In conclusion $C$. albicans is the only species from genus Candida isolated from oral lesion in this study, ethanol Ginkgo biloba extract have a good antifungal activity, higher number of phytochemical compounds and a higher antioxidant activity than aqueous extract.
\end{abstract}

Keywords: Candida albicans, Ginkgo biloba, Susceptibility test.

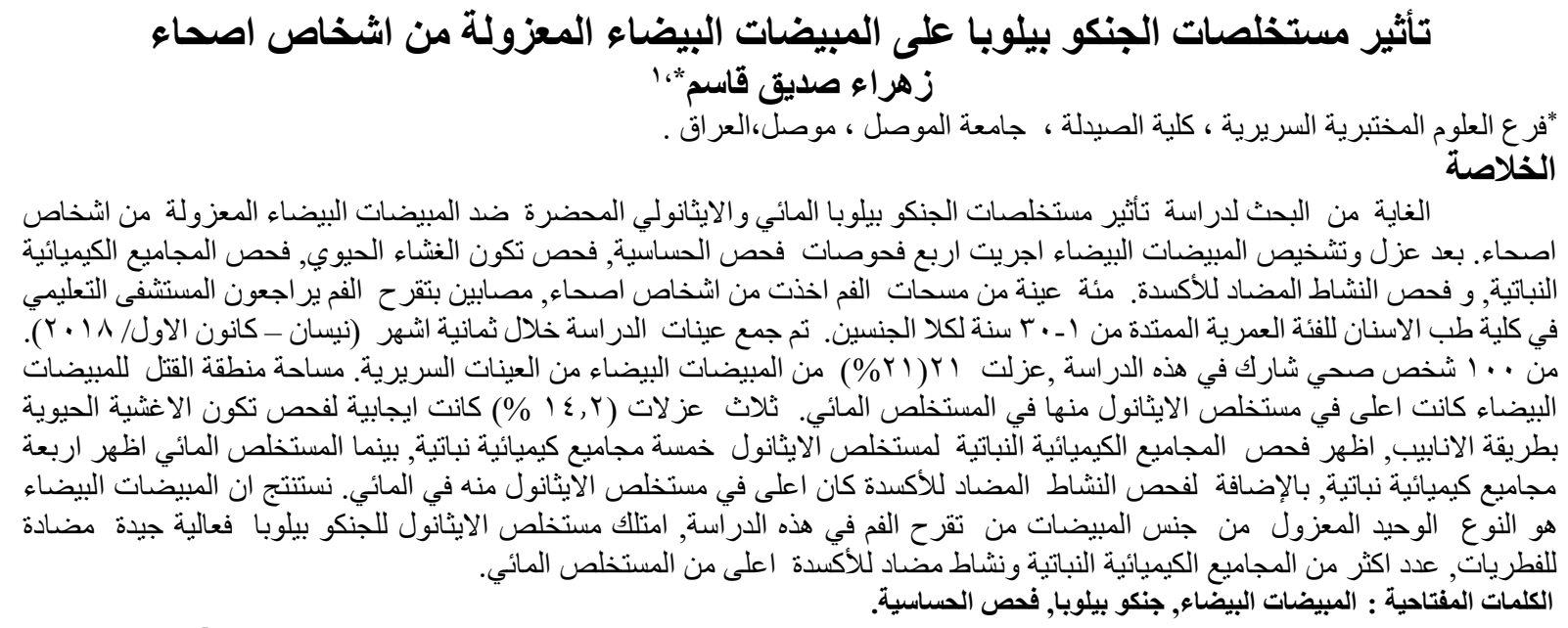

\section{Introduction}

A traditional Chinese medicine based on herbs has gained an increased acceptance from pharmaceutical companies as a rich resource for drug discovery ${ }^{(1)}$. Ginkgo biloba is the oldest living tree, and one of the most popular medicinal plants with a long history of use as a herbal medicine, its leaves extracts have been widely sold as a phytomedicine in Europe ${ }^{(1)}$. The effects of Ginkgo biloba extracts are due to its content of active phytochemicals compounds that possess pharmacological effects such as antiasthmatic, memory impairment and concentration difficulties improvement, wound healing, anticoagulant properties, and nerve cells protection ${ }^{(2)}$. The presence of phytochemicals in medicinal plants has been found a significant in the production of nonstandardized phytopharmaceutical products, the most important of these phytochemicals are alkaloids, tannins, flavonoids and phenolic compounds ${ }^{(3)}$.

Ginkgo biloba leaves extracts have $24 \%$ flavonoids, $6 \%$ terpenoids and less than $5 \mathrm{ppm}$ of natural antioxidants ginkgolic acids, this constitutes the most important active substances in the extract (4). Researchers are interested in antibacterial and antifungal effects that are obtained from medicinal plants sources with phytochemical activity, with advantage of no or less side effects that are often associated with synthetic antimicrobials ${ }^{(3)}$.

${ }^{1}$ Corresponding author E-mail: zahraasedeeq1980@ gmail.com

Received: $20 / 1 / 2020$

Accepted:20 / 5/2020

Iraqi Journal of Pharmaceutical Science 
Candida albicans is a member of genus Candida that is frequently isolated from mouth of healthy individuals and considered as a member of oral normal flora ${ }^{(5)}$. Oral cavity is a reservoir of more than 700 species of different microorganisms including Candida, which considered a normal colonizer in healthy and infected human mouth that resulting in different types of candidiasis ${ }^{(6)}$. In oral mucosal surface of healthy subjects Candida species resist the mechanical washing action of saliva, this resistance is an important factor for adherence and then biofilm formation which is one of the most virulence factors of C. albicans pathogenicity ${ }^{(5)}$.

Biofilm is structured of multiple cell types encased in an extracellular matrix, this biofilm phenotype of $C$. albicans has been shown to play a role in antifungal resistance (7). The rapid development of antifungal resistance by different Candida species has increased over the past decade, which associated with a rapid emergence of resistant strains of Candida species ${ }^{(8)}$. In addition, toxicity of the currently used antifungal agents has necessitated the need for alternative therapy, for these reasons, studies have been directed recently to focus on development of novel antifungal medicaments from plant resources ${ }^{(8)}$.

The aim of this research was to study the effect of prepared Ginkgo biloba (as aqueous and ethanolic) extracts against Candida albicans isolated from healthy persons, also to assess 4 tests for isolated C. albicans; susceptibility test, biofilm formation test, phytochemical screening test, and antioxidant activity test.

\section{Materials and Methods}

\section{Place and duration of the study}

This study included 100 oral swab samples obtained from oral cavity with lesion from healthy persons (of both sexes, age ranged from 1- 30 years) with bad oral hygiene attending dentistry teaching hospital, College of Dentistry, University of Mosul. Samples were collected over 8 months (April December / 2018).

\section{Isolation and identification $C$. albicans}

The samples were carried to the microbiology laboratory, College of Pharmacy, University of Mosul for isolation and identification. Each swab sample was cultured on Sabouraud dextrose agar. Chloramphenicol was added at a concentration of $0.05 \mathrm{~g} / \mathrm{L}$ to prevent any bacterial growth. Then the inoculated plates were incubated aerobically for $48-72$ hours at $37{ }^{\circ} \mathrm{C}$. Colonies of Candida on Sabouraud dextrose agar appeared as white creamy colonies. Further identification was applied including direct examination with Gram stain for the presence of budding Candida cells, and germ tube test which processed by transferring portion of pure cultured colony and mixed with 0.5 $\mathrm{ml}$ of human serum, then incubated at $37^{\circ} \mathrm{C}$ for $2-3$ hours, loopful of the inoculated serum examined for production of germ tube as short initial hyphae. In addition to chlamydospore formation test on cornmeal with tween 80 agar, this test was processed by inoculating cornmeal tween 80 agar with tested colony of Candida, then incubated the plates for 4872 hours at $37^{\circ} \mathrm{C}$, later on slide was prepared by lactophenol mount for production of chlamydospore (9,5). Microscopically examination of all isolated Candida by Gram stain, was done to confirm the identification of the $C$. albicans because it may confuse with the species $C$. dubliniensis which can give same results of tests mentioned above ${ }^{(10,11)}$.

\section{Preparation of Ginkgo biloba extracts}

The dried leaves of Ginkgo biloba were purchased from China, and converted into powder manually. Twenty five gram of dried Ginkgo biloba leaves were weighted and extracted with $250 \mathrm{ml}$ of ethanol in soxhlet extractor for 12 hours. For aqueous extraction 25 gram of Ginkgo biloba leaves powder were prepared by maceration with $250 \mathrm{ml}$ of sterile distilled water for 3 days at room temperature with frequent agitation. The aqueous and ethanol extracts were separately filtered with filter paper, then solvents were evaporate. The crude extracts were gummy and have dark yellow to green color (3). Finally the extracts of Ginkgo biloba leaves were kept freeze dried in refrigerator until use (1). Percentage yield of both extracts were calculated.

\section{Susceptibility test}

Susceptibility test was applied to each $C$. albicans isolates, which processed by transferring portion of pure culture colony of identified $C$. albicans and mixed with 3-5 $\mathrm{ml}$ Sabouraud broth to prepare a yeast suspension that equal to 0.5 MacFarland, then cotton swab was soaked in yeast suspension and streaked over a whole dried surface of Sabouraud dextrose agar plate ${ }^{(3)}$.

Preparation the discs

A stock concentration of $200 \mathrm{mg} / \mathrm{ml}$ was prepared for the aqueous and ethanol Ginkgo biloba extracts. The aqueous extract was dissolved in distilled water, while ethanol extract was dissolved in dimethyl sulfoxide (10\% DMSO) ${ }^{(12,13)}$. Paper discs (radius 6mm) prepared from sterile filter paper (Whatman No.1) were saturated with different concentrations $\quad(0.5 \mathrm{mg} / \mathrm{ml}, 1 \mathrm{mg} / \mathrm{ml}, 1.5 \mathrm{mg} / \mathrm{ml}$, $2 \mathrm{mg} / \mathrm{ml}, 2.5 \mathrm{mg} / \mathrm{ml}, 3 \mathrm{mg} / \mathrm{ml}$ ) of both Ginkgo biloba extracts overnight ${ }^{(3)}$. With a sterile forceps the discs were applied on the inoculated Sabouraud dextrose agar with $C$. albicans, then the plates were incubated for $24-48$ hours at $37^{\circ} \mathrm{C}$. The diameter of inhibition zone were measured in millimeter with a ruler in addition to the susceptibility of the isolates to control antifungal disc voriconazole lug. ${ }^{(14)}$.

A control isolate of Candida albicans (API 20 C system 6542114) used in this study, was obtained from microbiology laboratory, department of Clinical Laboratory Science, Collage of Pharmacy, University of Mosul. 


\section{Biofilm formation test}

This test was applied to each $C$. albicans isolates to test if they have an ability to form a biofilm or not by tube method. Ten $\mathrm{ml}$ of trypticase soy broth with $1 \%$ glucose inoculated with a portion of tested $C$. albicans colony in a test tube. The tubes were incubated at $37{ }^{\circ} \mathrm{C}$ for $16-18 \mathrm{~h}$. After incubation, tubes were decanted and washed with a normal saline and left to dry. Tubes were stained with crystal violet excess stain was washed with distilled water. Tubes were dried in inverted position. When a visible film lined the wall and bottom of the tube, biofilm formation was considered positive. The amount of biofilm formed was scored as none, weak, moderate, and high according to results of the control isolates ${ }^{(15)}$.

\section{Phytochemical screening test}

Phytochemical screening test used to detect the presence of phytochemicals including alkaloids, terpenoids, tannins, carbohydrates, saponin, coumarins glycosides, flavonoids, quinine, proteins. and steroids. These tests were applied separately to each Ginkgo biloba extracts. To detect the presence of phytochemicals according to standard procedures as reported by Soundararajan and Coworkers, 2017, and Kumar and Coworkers, $2018^{(16,17)}$.

1. Alkaloids: One ml of Mayer's reagent with a small amount of each extracts in a test tubes. The formation of yellow cream precipitate indicate the presence of alkaloid

2. Terpenoids: Two drops of chloroform, then one $\mathrm{ml}$ of concentrated hydrochloric acid was added to a small amount of each extracts, then heated for two minutes. A reddish brown color was formed to show positive results for the presence of terpenoids.

3. Tannins: Few drops of $10 \%$ neutral lead acetate solution were added to a small amount of each extracts. White to pale yellow precipitate Formation indicates the presence of tannins.

4. Carbohydrates : Small amount of alcoholic $\alpha$ naphthol solution in a test tube, then concentrated sulphuric acid was added carefully along the side of the test tube, and a small amount of each extracts were added. Violet ring formation at the junction indicated the presence of carbohydrates.

5. Saponin: Two to three drops of distilled water was added to a small amount of each extracts and shaking well. Semi perminant foam formation indicate the presence of saponin.

6. Coumarins glycosides: Diluted $\mathrm{HNO}_{3}$ was added to a small amount of each extracts. The presence of coumarins glycosides was indicated by the presence of yellow precipitate ${ }^{(17)}$.

7. Flavonoids: Few drops of $\mathrm{FeCL}_{3}$ solution were added to a small amount of each extracts. Green to black color formation indicates the presence of flavonoids. Phenolic compounds not necessarily flavonoids.

8. Quinine: Small amount of each extracts were added to $1 \mathrm{ml}$ sodium hydroxide solution a and kept for 2 minute. Forming reddish brown color indicate the presence of quinine.

9. Proteins: One drop of concentrated nitric acid was added to a small amount of each extracts. Formation reddish color indicate the presence of proteins.

10. Steroids: Small amount of each extracts were added to one $\mathrm{ml}$ of chloroform, and $1 \mathrm{ml}$ of concentrated sulphuric acid, then shaken well and allowed to stand for five minutes. Red color appearance in the lower layer indicates the presence of steroids ${ }^{(16)}$.

\section{Antioxidant activity test}

This test is based on the scavenging activity of the stable 2,2-diphenyl-1-picrylhydrazyl (DPPH) free radicals, with the antioxidant activity of Ginkgo biloba extracts ${ }^{(18)}$.This test was applied separately to each Ginkgo biloba extracts, which prepared by taking a three volumes (50-100-150 ul) of each Ginkgo biloba extracts were added to test tubes then completed the volume to $(1 \mathrm{ml})$ by distilled water (D.W), then $1 \mathrm{ml}$ of DPPH solution was added to each tube, mixed well and incubated the test tubes at room temperature for $30 \mathrm{~min}$. Control was prepared from ascorbic acid solution $(0.03 \% \mathrm{~W} / \mathrm{V})$. The absorbance (A) was measured at $517 \mathrm{~nm}$ using (spectrophotometer). Calculation the inhibition of DPPH free radical in percent $(1 \%)$ was from the following equation ${ }^{(19)} \quad 1 \%=\left\{\left(\mathrm{A}_{\mathrm{c}}\right.\right.$ - $\left.\left.\mathrm{A}_{\mathrm{s}}\right) / \mathrm{A}_{\mathrm{c}}\right\} * 100$

$\mathrm{A}_{\mathrm{c}}$ : The absorbance of the control

$\mathrm{A}_{\mathrm{s}}$ : The absorbance of the sample

Statistical analysis: number, mean, part per million, percentage.

\section{Results and Discussion}

From 100 healthy persons with oral lesion, involved in this study there were 42 males and 58 females. Twenty one healthy person $(21 \%)$ showed positive results for presence of $C$. albicans in their oral swab samples, $12(12 \%)$ were females while $9(9 \%)$ were males (Table1). Different tests were used to identify the isolated C.albicans as Gram stain, germ tube test, and chlamydospore formation test as shown in (Figure 1). The effect obtained from aqueous and ethanol extracts of Ginkgo biloba on the growth of Candida albicans and control isolates, was nearly similar. 


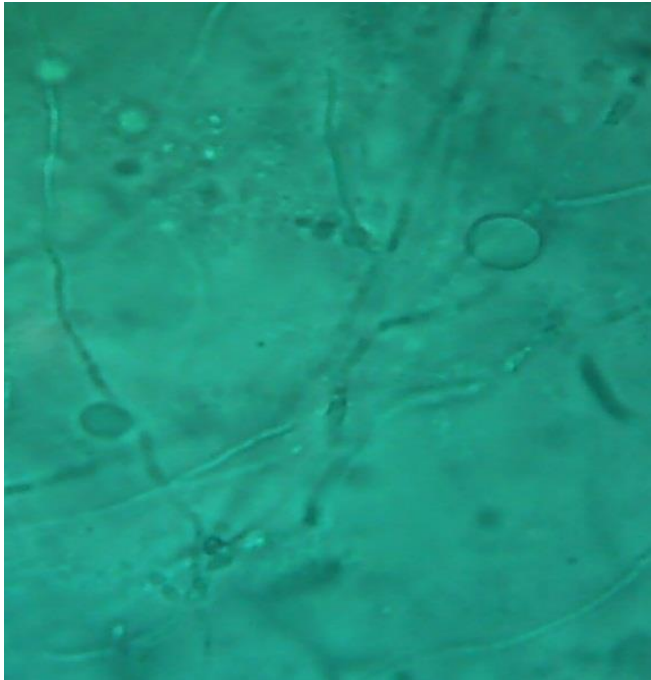

Figure 1. Chlamydospore of $C$. albicans by lactophenol mount.

Candida albicans is the main species among 20 species of genus Candida inhabiting the oral cavity. It is estimated that oral candidal carriage accounts for about $35 \%-80 \%$ of all oral yeast isolates ${ }^{(20)}$. The percentage of C.albicans infection in this study was $(21 \%)$, Zaremba and Coworkers, 2006 found that $C$. albicans isolates in the oral cavity of adults with and without dentures were $17 / 32 ; 53.1 \%$ and $38 / 71 ; 53.5 \%$, respectively ${ }^{(21)}$. However the percentage of $C$. albicans isolated in this study was higher in females than males $(12 \%$ vs. $9 \%$ respectively). In addition to that the number of isolated C. albicans was found to increase with age of the studied subjects (Table1). This might be due to the fact that the number of females involved at the time of this study was higher than males. In accordance to this finding, the frequent carriers of Candida species were found to be higher in females than males and increased with age ${ }^{(6)}$. However, Blazi and Coworkers, $2005^{(22)}$ did not detect any association between age and candidal growth in between diabetic patients and healthy subjects. Every 25 gram of Ginkgo biloba leaves extract yielded $6.2(24.8 \%)$ gram for aqueous and 3.8 $(15.2 \%)$ gram for ethanol extract as shown in Table 2.

Table 1. The presence of $C$. albicans in male and female according to their age.

\begin{tabular}{|c|c|c|c|c|c|c|}
\hline \multirow{3}{*}{$\begin{array}{l}\text { Age of healthy } \\
\text { persons }\end{array}$} & \multicolumn{2}{|c|}{ sex } & \multicolumn{4}{|c|}{ Candida albicans } \\
\hline & \multirow{2}{*}{$\begin{array}{c}\text { Male } \\
\text { No. }\end{array}$} & \multirow{2}{*}{$\begin{array}{c}\text { Female } \\
\text { No. }\end{array}$} & \multicolumn{2}{|c|}{ Male } & \multicolumn{2}{|c|}{ Female } \\
\hline & & & No. & $\%$ & No. & $\%$ \\
\hline $1-5$ & 0 & 2 & 0 & 0 & 0 & 0 \\
\hline $6-10$ & 4 & 7 & 1 & 1 & 0 & 0 \\
\hline 11-15 & 7 & 10 & 1 & 1 & 2 & 2 \\
\hline $16-20$ & 8 & 11 & 2 & 2 & 3 & 3 \\
\hline $21-25$ & 10 & 13 & 2 & 2 & 3 & 3 \\
\hline $26-30$ & 13 & 15 & 3 & 3 & 4 & 4 \\
\hline Total & 42 & 58 & 9 & 9 & 12 & 12 \\
\hline
\end{tabular}

Table 2. Weight and percentage of aqueous and ethanol of extracts from 25 gram Ginkgo biloba leaves.

\begin{tabular}{|c|c|c|c|}
\hline \multicolumn{2}{|c|}{ Aqueous extract of Ginkgo biloba } & \multicolumn{2}{c|}{ Ethanol extract of Ginkgo biloba } \\
\hline Wt.(gram) & $\%$ & Wt.(gram) & $\%$ \\
\hline 6.2 & 24.8 & 3.8 & 15.2 \\
\hline
\end{tabular}

\section{Phytochemical screening test}

The phytochemical screening test of both aqueous and ethanol extract was performed separately for each extract. The results of this test are shown in (Table 3). In ethanol extract the phytochemical reactions were positive for alkaloids, flavonoids, tannin, carbohydrate, and coumarins glycosides. However, detection of terpenoids, saponine, quinine, proteins, steroids showed negative result. Flavonoids, tannins, carbohydrates, and proteins were detected in the aqueous extract while the test did not reveal any positive finding for alkaloids, terpenoids, saponine, quinine, steroids, and coumarins glycosides.

The lower antimicrobial activity of the aqueous extract might be due to the less phytochemicals (flavonoids, tannins, carbohydrate, and protein) obtained by the aqueous extraction in comparison to the alcoholic extraction. In the current study, higher number of phytochemicals was present in ethanol extract than aqueous. This explain the largest zone of inhibition for ethanol extract in antifungal susceptibility test, due to fact that ethanol is the best solvent for the active compounds extracted from plants, compared with a distilled water used as a solvent in the aqueous extract. Similarly, Ibrahim and Coworkers, 2016 extracted (glycosides, saponines, steroids, triterpenes, tannins, flavonoids, anthraquinones, and alkaloids), these constituents have been shown to be responsible for the pharmacological activities against Staphylococcus aureus, Klebsiella pneumoniae, Escherichia coli, C. albicans, Saccharomyces cerevisiae, and Geotrichum 
candidum with zone of inhibition ranging from 22$27 \mathrm{~mm}$. This finding is consistent with that Ginkgo biloba extracts contained active compounds that showed considerable activity on bacteria and fungi (23).

Table 3. Phytochemical screening test of both Ginkgo biloba extracts.

\begin{tabular}{|c|c|c|c|c|c|c|c|c|c|c|}
\hline \multirow[b]{2}{*}{ Type of extracts } & \multicolumn{10}{|c|}{ Phytochemical reactions } \\
\hline & Alk. & Terp & Fla. & Tan. & Carb. & Sap. & Qui. & Pro. & St. & Cou. \\
\hline Aqueous & - & - & + & + & + & - & - & + & - & - \\
\hline Ethanol & + & - & + & + & + & - & - & - & - & + \\
\hline
\end{tabular}

\section{Susceptibility test}

This test was applied to 21 C. albicans isolates by using the two types (aqueous and ethanol) of prepared Ginkgo biloba extracts with different concentrations. Five isolates were shown resistant to all concentrations of Ginkgo biloba both extracts. Sixteen isolates were sensitive to both aqueous and ethanol extracts. The mean results of susceptibility test zone diameter indicated that Ginkgo biloba ethanol extract showed a higher zone of inhibition than aqueous as showed in (Table 4 and Figure $2 \mathrm{~A}, 2 \mathrm{~B}$ ).

The susceptibility test of Ginkgo biloba extracts was investigated using disc agar diffusion method. Sixteen $C$. albicans isolates were found susceptible to both Ginkgo biloba extracts. The anticandidal effect of the prepared extracts was concentration dependent. The results showed a good antifungal activity of both Ginkgo biloba extracts with zone inhibition ranging from $8-22 \mathrm{~mm}$ for aqueous extract and 10-25 $\mathrm{mm}$ for ethanol extract (Table 4 and Figures 2A, 2B). Form the results obtained in our study ethanol extract had a higher growth inhibitory effects on $C$. albicans than aqueous extract, this might be due to the presence of a variety of active phytochemical compounds in the ethanol extract as alkaloids, flavonoids, tannins, carbohydrate, and coumarine glycosides.

Table 4. The mean diameter of inhibition zone in millimeter against different concentrations of both Ginkgo biloba extracts and voriconazole as a control.

\begin{tabular}{|c|c|c|c|c|c|c|c|}
\hline \multirow{3}{*}{ Type of extracts } & \multicolumn{6}{|c|}{ Concentration in $\mathrm{mg} / \mathrm{ml}$ of extract } & \multirow{2}{*}{$\begin{array}{r}\begin{array}{c}\text { Voriconazole } \\
\text { as control }\end{array} \\
1 \mathrm{ug}\end{array}$} \\
\hline & 0.5 & 1 & 1.5 & 2 & 2.5 & 3 & \\
\hline & \multicolumn{7}{|c|}{ Mean zone diameter in (mm) } \\
\hline Aqueous extract & 8 & 10 & 12 & 14 & 18 & 22 & 20 \\
\hline Ethanol extract & 10 & 14 & 18 & 20 & 25 & 25 & 22 \\
\hline
\end{tabular}

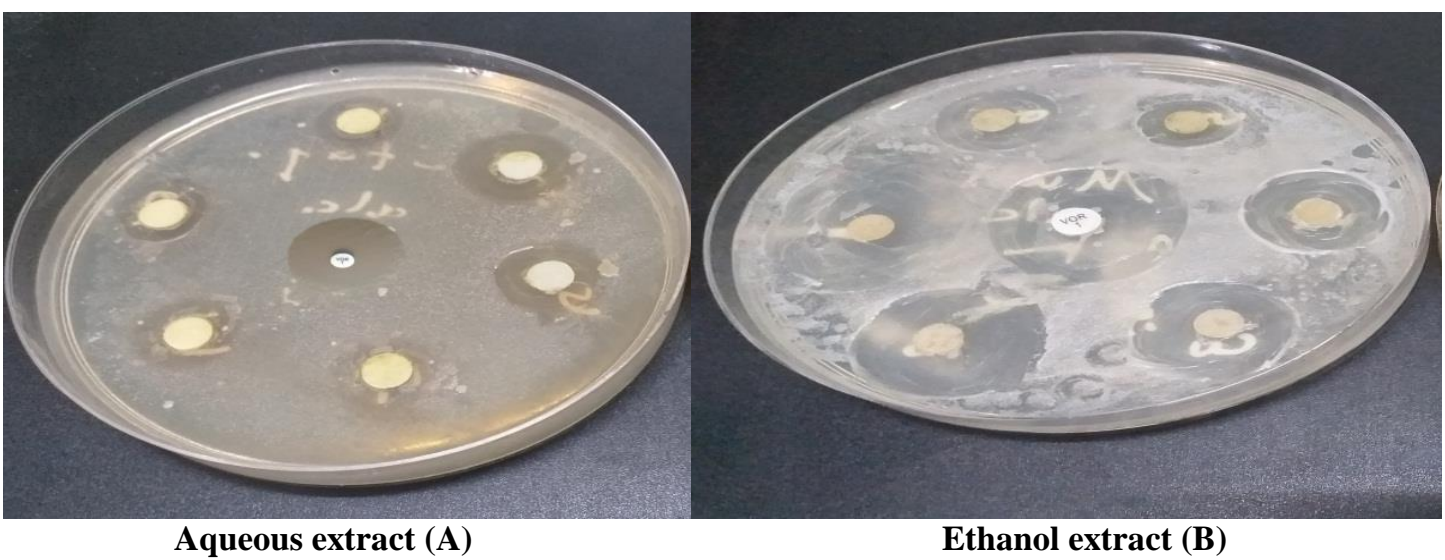

Figure 2. The susceptibility test of Ginkgo biloba extracts against $C$. albicans.

\section{Biofilm formation test}

Among the $21(21 \%)$ C. albicans isolates there were $3(14.2 \%)$ of them showed the ability to form a biofilm in tube method. One $(4.7 \%)$ isolate showed weak biofilm formation indicated by faint staining, while the other $2(9.5 \%)$ isolates were 
moderate biofilm former. Eighteen $(85.7 \%)$ of the isolates didn't form a biofilm as showed in (Table 5).

Biofilm formation represents a significant clinical challenge in the management of microbial infections ${ }^{(15)}$. The major virulence attributed to $C$. albicans has been shown to belong to its ability to adhere to oral epithelium then form a biofilm, these biofilm is resistant to conventional antifungal therapy ${ }^{(8)}$. Tube method is $73 \%$ sensitive, $92.5 \%$ specific and $80 \%$ accurate for biofilm detection ${ }^{(15)}$.

Table 5. Number and percentage of the isolates that formation biofilm by tube method.

\begin{tabular}{||c|c|c|c||}
\hline \multirow{4}{*}{ Number of isolates (21) } & Biofilm formation & \multicolumn{2}{|c|}{ Tube method } \\
\cline { 2 - 4 } & High & 0 & 0 \\
\cline { 2 - 4 } & Moderate & 2 & 9.5 \\
\cline { 2 - 4 } & Weak & 1 & 4.7 \\
\cline { 2 - 4 } & None & 18 & 85.7 \\
\hline
\end{tabular}

\section{Antioxidant activity test}

The reduction capability of DPPH radical is determined by the decrease in absorbance at 517 $\mathrm{nm}$ induced by antioxidants activity present in both Ginkgo biloba extracts. Data indicated that the both extracts are able to reduce the stable radical DPPH to yellow colored. The scavenging effect at high concentration (6107.14) of both extracts and ascorbic acid solution was in the following order. Ethanol extract $>$ ascorbic acid $>$ aqueous extract as showed in Table 6.

The antioxidant activity of Ginkgo biloba extracts is attributed to their contents of antioxidant agents, these antioxidants have a redox properties which play an important role in adsorbing and neutralizing free radicals ${ }^{(19)}$. Data showed that the scavenging activity of both extracts were increased with increasing in the concentration of each extract, ethanol extract in all concentrations showed a higher antioxidant activity than aqueous extract, this could be due to the fact that ethanol extract have a highly content of total phenolic and flavonoids than aqueous ${ }^{(19)}$. The antioxidant activity of ethanol extract at concentration $6107.14 \mathrm{ppm}$ showed a higher scavenging activity than 23 ppm of ascorbic acid, on the other hand the scavenging activity of aqueous extract at concentration 6107.14 was lower than 23 ppm ascorbic acid.

Constituents of plant materials that have antioxidant activity is very important in the maintenance of health and protection from diseases (19). Ginkgo biloba may have chemopreventive anticancer properties that related to their antioxidant activity, anti-angiogenic and gene -regulatory action $^{(24)}$.

Table 6. The antioxidant activity of Ginkgo biloba aqueous, ethanol and ascorbic acid against DPPH radicals.

\begin{tabular}{||c|c|c||}
\hline \hline Extract & Concentration $(\mathbf{p p m})$ & Scavenging activity \% \\
\hline \multirow{3}{*}{ Aqueous extract } & 2035.71 & 66.60 \\
\cline { 2 - 3 } & 4071.41 & 80.70 \\
\cline { 2 - 3 } & 6107.14 & 82.16 \\
\hline \multirow{3}{*}{ Ethanol extract } & 2035.71 & 62.01 \\
\cline { 2 - 3 } & 4071.41 & 94.20 \\
\cline { 2 - 3 } & 6107.14 & 96.02 \\
\hline Ascorbic acid & 23 & 88.15 \\
\hline
\end{tabular}

\section{Conclusion}

The results of this study showed that ethanolic extract of Ginkgo biloba leaves, have a good antifungal, phytochemicals, and antioxidant activity. Gingko biloba extract can be used in pharmaceutical herbal medicinal oral product as a tooth paste, oral wash or any other oral medication.

\section{Acknowledgement}

The author is grateful to acknowledge the College of Pharmacy - University of Mosul for providing the necessary facilities to carry out this study.

\section{References}

1. Bahri G, Lamuki M, Rezae-Raad M. Antiproliferative effects of alcoholic and aqueous extract of Ginkgo biloba green leaves on MCF7 cell line. Inter J Pharmaceutical Med Res. 2014; 2(3):8-11.

2. Rimkiene L, Kubiliene A, Zevzikovas A, Kazlauskiene D, Jakstas V. Variation in flavonoid composition and radical - scavenging activity in Ginkgo biloba L.due to the growth location and time of harvest. J Food Quality. 2017; 1-8. 
3. Punnagai K, Darling Chellathai D, Karthik VP, Glory Josephine I. Evaluation of antifungal activity of ethanolic extract of andrographis echioides - an in vitro study. Inter J Pharm Bio Sci. 2016; 7(4): 6-10.

4. Zhang N, Lan W, Wang Q, Sun X, Xie J. Antibacterial mechanism of Ginkgo biloba leaf extract when applied to Shewanella putrefaciens and Saprophytic Staphylococcus. Aquaculture Fisheries. 2018;3(4) :163-169.

5. Williams D, Lewis $M$. Isolation and identification of Candida from oral cavity. Oral Dis. 2000; 6(1): 3-11.

6. Prakash B, Shekar M, Maiti B, Karunasagar I, Padiyath S. Prevalence of Candida spp. among healthy denture and nondenture wearers with respect to hygiene and age. J Indian Prosth Society. 2015;15(1):29-32.

7. Sitheeque M, Samaranayake L. Chronic hyperplastic candidosis/candidiasis (candidal leukoplakia). Crit Rev Oral Biol Med. 2003;14(4): 253-267.

8. Gulati M, Nobile C. Candida albicans biofilms: development, regulation, and molecular mechanisms. Microbes infect. 2016;18(5):310321.

9. Axell T, Simonsson T, Birkhed D, Rosenborg J, Edwardsson S. Evaluation of a simplified diagnostic aid (Oricult-N) for detection of oral candidoses . Scandinavian J Dent Res. 1985; 93(1): 52-55.

10. McCullough M, Clemons $K$, Stevens D. Molecular and phenotypic characterization of genotypic Candida albicans subgroups and comparison with Candida dubliniensis and Candida stellatoidea. J Clin Microbiol. 1999; 37(2):417-421.

11. Vilela S, Kamei K, Sano A, Tanaka R, Uno J, Takahashi I, et al. pathogenicity and virulence of Candida dubliniensis: comparison with $C$. albicans. Med Mycol. 2002; 40: 249-257.

12. Shihabudeen M, Priscilla H, Thirumurugan D. Antimicrobial activity and phytochemical analysis of selected Indian folk medicinal plants. Inter J Pharma Sci Res (IJPSR). 2010;1(10):430-434.

13. Jameela $M$, Mohideen A, Sunitha $K$, Narayanan M. Antibacterial activities of three medicinal plant extract against fish pathogens. Inter J Biol Tech. 2011;2(2):57-60.

14. Jouda M, Elbashiti $T$, Masad A. The antibacterial effect of some medicinal plant extracts and their synergistic effect with antibiotics. Adv Life Sci Tech. 2016;46:59 -69.

15. Hassan A, Usman J, Kaleem F, Omair M, Khalid A, Iqbal M. Evaluation of different detection methods of biofilm formation in the clinical isolates. Braz J Infect Dis. 2011;15(4): 305-311.

16. Soundararajan G, Ramesh Babu N G, Johney J, Ragunathan R. Extraction of bioactive compounds from rosmarinus officinalis L. and its anticancer activity against HeLa cell line. Inter J Sci Res (IJSR).2017; 6(8). 165-168.

17. Kumar N, Upadhyay P, Saxena G. Phytochemical screening of pomegranate peel using crude hydro-alcoholic extract and pharmacological activities . J Sci Res Publications. 2018; 8(1):193 -199.

18. Lee J, Hwang W, Lim S. Antioxidant and anticancer activities of organic extracts from platycodon grandiflorum A de Candolle roots. J Ethnopharmacol. 2004;93(2-3):409-415.

19. Hassan $H$, Hassan N. In vitro antioxidant and free radical scavenging activities of red grape seed extracts. Global J Biotech \& Biochem. 2010;5(2): 106-115.

20. Scully C, Ei-Kabir M, Samaranayake L. Candida and oral candidosis: A review. Crit Rev Oral Biol Med. 1994;5(2): 125-157.

21. Zaremba M, Daniluk T, Rozkiewicz D, CylwikRokicka D, Kierklo A, Tokajuk G, et al. Incidence rate of Candida species in the oral cavity of middle - aged and elderly subjects. Adv Med Sci. 2006;51(1): 233-236.

22. Belazi M, Velegraki A, Fleva A, Gidarakou I, Papanaum L, Baka D, et al. Candidal overgrowth in diabetic patients: potential predisposing. Mycoses. 2005;48(3):192-196

23. Ibrahim M, Nuhu A. Phytochemical screening and antibacterial/antifungal activities of Ginkgo biloba extract EGb 761. J Pharm Biol Sci (JOSR-JPBS) .2016;11(1): 43-49.

24. DeFeudis F, Papadopoulos V, Drieu K. Ginkgo biloba extracts and cancer: a research area in infancy. Fundam Clin Pharmacol. 2003;17(4): 405-417. 